\title{
First Data from the TOTEM experiment at LHC
}

\author{
F. Cafagna* for the TOTEM Collaboration \\ INFN, Bari unit \\ E-mail: Erancesco.cafagna@ba.infn.it
}

Totem is the only LHC experiment that will explore the forward region at pseudorapidity larger than 3.1. The main goal is the measurement of the total and elastic cross-section at $14 \mathrm{TeV}$ and the study of diffractive physics in the forward region. The experiment approved and funded in the 2006, was build, largely commissioned and started his data taking in December 2009. The total cross section beyond $1 \mathrm{TeV} / c$ will be measured with the unprecedent precision of $1 \%$ using the luminosity independent method based on the simultaneous detection of elastic scattering at low momentum transfer and of the inelastic interactions. Protons scattered at very small angles in elastic or quasi-elastic reactions will be measured in telescopes of silicon detectors enclosed in Roman Pots, placed on both sides of the intersection regions. Inelastically produced secondaries will be measured by a forward inelastic detector covering the region $3<\eta<7$ with full azimutal acceptance. This last detector will measure the overall rate of inelastic reactions. The TOTEM physics program also include for the first time the measurement of the charged multiplicity at the $\mathrm{TeV}$ scale important for the understanding of the cosmic ray events. TOTEM will take data under all LHC beam conditions including standard high luminosity runs to maximize its physics goals. This contribution describes the status of the TOTEM experiment. A first set of data at 0.9 and $2.36 \mathrm{TeV}$ was recently collected and some preliminary results will be shown. In addition we will discuss the measurements to be made in the 2010 LHC runs.

35th International Conference of High Energy Physics - ICHEP2010,

July 22-28, 2010

Paris France

${ }^{*}$ Speaker. 


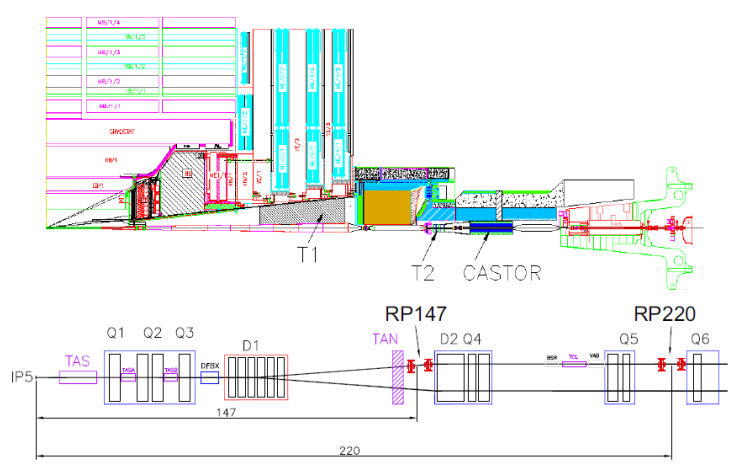

Figure 1: The TOTEM experiment layout. The position of the forward telescopes $\mathrm{T} 1$ and $\mathrm{T} 2$ in the CMS experiment (top) and of the RPs, along the LHC beam line, at 147m (RP147) and 220m (RP220) (bottom).

\section{Introduction}

The TOTEM experiment[1] 2] is dedicated to the measurement of the total proton-proton crosssection with the luminosity-independent method based on the Optical Theorem. This method requires a precise knowledge of the elastic scattering cross-section down to a squared four-momentum transfer of $|t| \sim 10^{-3} \mathrm{GeV}^{2}$ along with the measurement of the total inelastic rate. Furthermore, TOTEM's physics program aims at a deeper understanding of the proton structure by studying elastic scattering with large momentum transfers, and via a comprehensive menu of diffractive processes. To fulfill these objectives the TOTEM collaboration had to invest heavily in the design of detectors that will be capable of meeting the challenge of triggering and recording events in the very forward region. TOTEM's unprecedented coverage in the pseudo-rapidity range of $3.1 \leq|\eta| \leq 6.5\left(\eta=-\ln \left(\tan \left(\frac{\theta}{2}\right)\right)\right)$ on both sides of the interaction point is accomplished by two telescopes for inelastically produced charged particles, so called T1 and T2, installed inside the CMS detector at the interaction point IP5. These telescopes are complemented by detectors in special movable beam-pipe insertions, the so called Roman Pot (RP) detectors, placed at about $147 \mathrm{~m}$ and $220 \mathrm{~m}$ from the interaction point, designed to detect leading protons at merely a few $\mathrm{mm}$ from the beam center. In Figure 1 T1, T2 and RP positions in CMS and along the LHC tunnel are shown.

\section{TOTEM detectors}

The $\mathrm{T} 1$ and $\mathrm{T} 2$ telescopes trigger and partially reconstruct inelastic events. Together they must not only provide an inclusive trigger for diffractive events but also reconstruct their vertex.

The T1 detector[抣, is the inelastic telescope closest to the interaction point. It is centered at $\mathrm{z}$ $=9 \mathrm{~m}$ and installed in two cone-shaped regions in the endcaps of CMS. Each arm of the telescope, is composed of five planes of Cathode Strip Chambers (CSC), with six chambers in each plane covering roughly a region of $60^{\circ}$ in $\phi$. Each arm is subdivided into two quarter, one quarter of T1 is shown in Figure 4 . In every plane the CSC readout strips of the two cathode planes are oriented at $60^{\circ}$ with respect to the anode wires. This geometry allows the measurement of three coordinates for each particle track, which significantly helps in resolving multiple events. To improve pattern recognition, the planes are rotated by $3^{\circ}$ with respect to each other. 
The second telescope, T2[2, 田, it is located in two opposite position, centered at $\pm 13.5 \mathrm{~m}$ respect to the interaction point. The T2 detectors exploit the usage of triple Gas Electron Multipliers (GEM). GEMs are gas-filled detectors combining a good spatial resolution along with a very high rate capability and a good resistance to radiation. Each of the two telescope arms is made of two sets of 10 aligned detector planes with semicircular shape, mounted on each side of the vacuum pipe. To avoid efficiency losses on the boundaries, the angular coverage of each half plane is larger than $180^{\circ}$. The readout of the half planes has two separate layers with different patterns: one with 256 concentric rings, $80 \mu \mathrm{m}$ wide and with a pitch of $400 \mu \mathrm{m}$, providing the radial coordinates of traversing tracks with a good precision, and the other with a matrix of 1536 pads varying in size from $2 \times 2 \mathrm{~mm}^{2}$ to $7 \times 7 \mathrm{~mm}^{2}$. A picture of one of the $\mathrm{T} 2$ arms during the installation, along with the one of a single GEM plane, is shown in Figure 3 .

High efficiency up to the physical detector border is an essential feature to maximize acceptance for protons scattered elastically or diffractively at polar angles down to a few micro radians at the interaction point. The RP station special beam pipe insertion mechanism, allows bringing the detectors very close to the beam. Each RP station[2] is made of two units separated by $4 \mathrm{~m}$ and equipped with one horizontal and two vertical pots. In each pot, a detector package made of 10 planes of single-sided silicon micro-strip detectors, is inserted. These are planar edgeless silicon detectors with current-terminating structure (CTS)[3] designed by TOTEM with the specific objective of reducing the insensitive area, at the edge facing the beam, to only a few tens of microns. Sensors have a diamond-like shape and are capable of precision of less than $20 \mu \mathrm{m}$ thanks to a strip pitch of $66 \mu \mathrm{m}$. Strips are oriented at $45^{\circ}$ with respect to the side of the sensor facing the beam. Sensors are mounted back-to-back in pairs in order to have their strips oriented mutually orthogonal. Each detector package is operated at $-30^{\circ} \mathrm{C}$ and is kept in a controlled atmosphere with pressure between 10mbar and 100mbar. A RP unit and a detector package is shown in Figure 2 .

The read-out of all TOTEM subsystems is based on the custom developed digital VFAT chip with trigger capability. The data acquisition system is designed to be compatible with the CMS DAQ to make common data taking possible at a later stage.

\section{Status and perspectives}

During the LHC 2010 runs, the full T2 detector and the RP stations at 220m where installed, commissioned and taking data for physics at $7 \mathrm{TeV}$. Data have been collected with both detectors and the mechanism of RP insertion has been commissioned and qualified. At the time of the
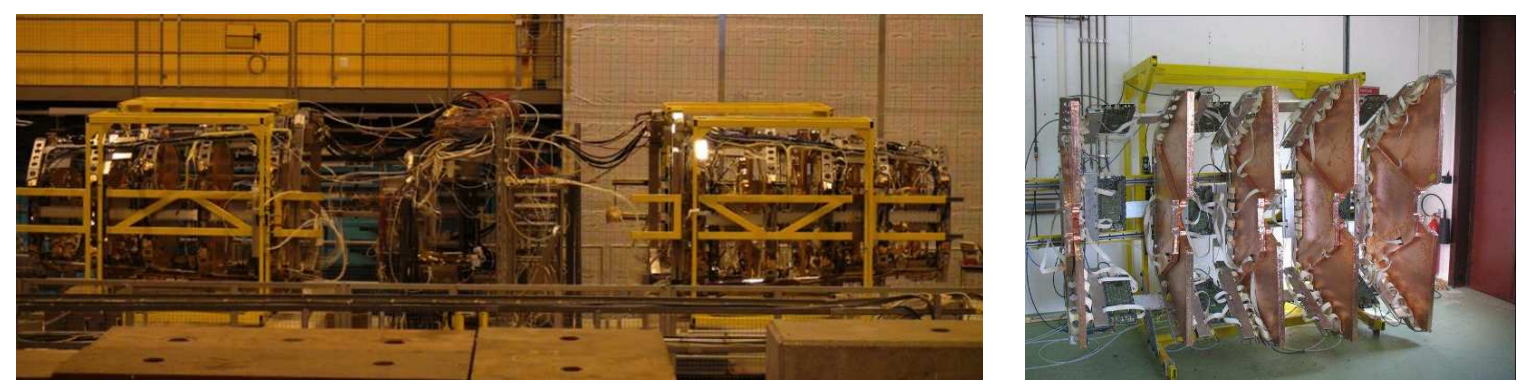

Figure 2: The full T1 detector during the beam tests in the SPS experimental area (left) and a quarter (right). 

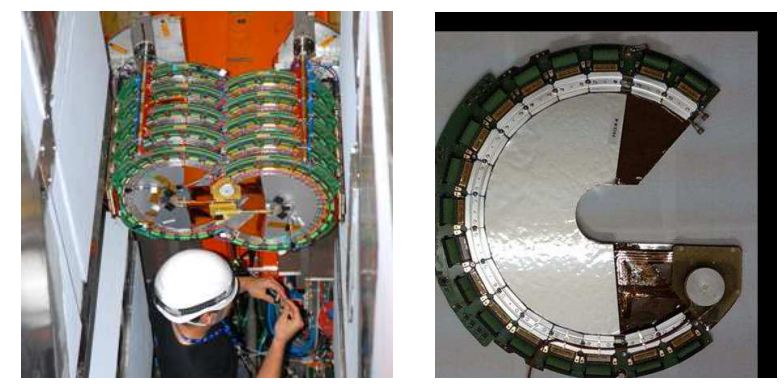

Figure 3: One half of the T2 during the installation (left) and one plane of GEM fully assembled (right).

conference, statistic has been collected with RP inserted at 25 and $20 \sigma$ allowing the alignment of the detectors and the investigation of the beam parameters as well as the estimation of the detector efficiencies and backgrounds. In the meantime $\mathrm{T} 1$ detector is fully operational in the beam test area of the SPS facility, waiting to be integrated during the LHC winter stop, and the construction and test of the RP stations, to be placed at $147 \mathrm{~m}$, are in progress.

The precise measurements of the total cross sections, up to $\sim 1 \%$, require a $\beta^{*}$ as high as $1540 \mathrm{~m}$ to maximize the acceptance for elastic protons. With a $\beta^{*}$ of $90 \mathrm{~m}$ the precision of the measurement will scale to $\sim 5-6 \%$. The availability of such a special optic depends on the machine status and schedule, in the mean time, with the RPs detectors inserted at their nominal position, TOTEM it is ready to investigate its physics program at low $\beta^{*}$, that is: forward charge particles studies, large $|t|$ elastic scattering and the study of high mass single and central diffraction.

\section{References}

[1] V. Berardi et al., Totel cross-section, elastic scattering and diffraction dissociation at the Large Hadron Collider at CERN: TOTEM technical design report, CERN-LHCC-2004-002; Addendum CERN-LHCC-2004-020.

[2] G. Anelli et al., The TOTEM Experiment at the CERN Large Hadron Collider, 2008 JINST 3 S08007.

[3] G. Ruggiero et al., Characteristics of edgeless silicon detectors for the Roman Pots of the TOTEM experiment at the LHC, Nucl.Instrum.Meth. A604 (2009) 242-245

[4] S. Lami et al., A triple-GEM telescope for the TOTEM experiment, Nucl. Phys. B, Proc. Suppl. 172 (2007) 231-233
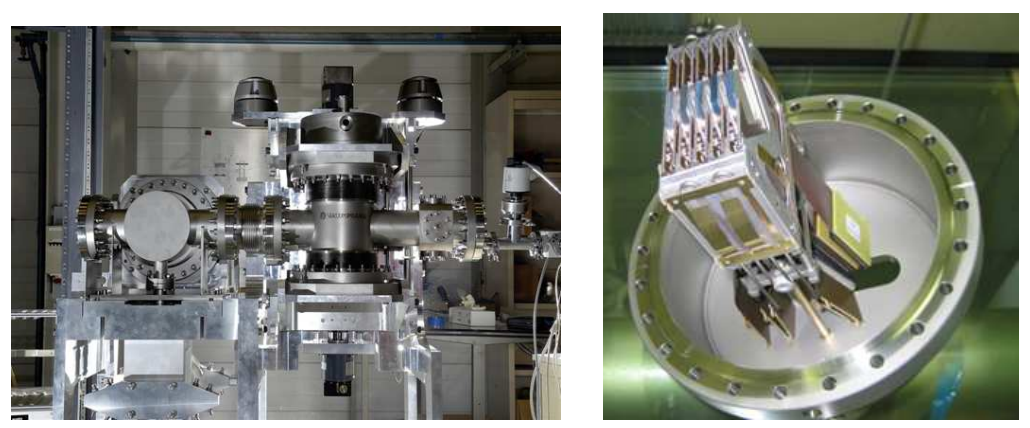

Figure 4: One of the two Roman Pot station installed at 220m from the IP5 interaction point (left). A detail of one pot fine silicon planes, made of ten detectors, is shown as well (right) 